\title{
Evaluación de los efectos del Programa de Apoyos Directos al Campo (Procampo) en el mercado de maíz en México, 2005-2007
}

\section{Evaluation of the effects of Programa de Apoyos Directos al Campo (Procampo) on the market of maize in Mexico, 2005-2007}

\author{
José Alberto García-Salazar* \\ RHONDA K. SKaGgs** \\ Terry L. CraWford**
}

\begin{abstract}
The Program of Countryside Funding (Programa de Apoyos al Campo, Procampo) is a direct funding to the income of producers that started in 1993. Maize is the cultivation which has obtained the most resources from Procampo. The results of a spatial and inter-temporal equilibrium model indicate that in the absence of Procampo, the yearly production and consumption would have been 18.7 and 28.8 million tons in the 2005-2007 period. The benefits were higher than the costs of the program, and an increment of $50 \%$ in subsidy would increase maize production in 1.6 million metric tons and would decrease imports in 20.7\%. From the positive effects of the Program, its continuation is recommended, as well as the increment of the funding.
\end{abstract}

Keywords: maize, Procampo, producer and consumer surplus, importations, spatial and inter-temporary equilibrium model.

\section{Resumen}

El Programa de Apoyos Directos al Campo (Procampo) es un pago directo al ingreso de los productores de México que inició en 1993. El maíz es el cultivo que más recursos ha obtenido de Procampo. Los resultados de un modelo de equilibrio espacial e intertemporal indican que en ausencia de Procampo, la producción y el consumo anual de maíz hubieran sido 18.7 y 28.8 millones de toneladas en el periodo 2005-2007. Los beneficios fueron más altos que los costos del programa, y un aumento de $50 \%$ en el subsidio aumentaría la producción de maíz en 1.6 millones de toneladas y disminuiría las importaciones en $20.7 \%$. Debido a los efectos positivos del Programa, se recomienda la continuación y el aumento del apoyo.

Palabras clave: maíz, Procampo, excedentes al productor y consumidor, importaciones, modelo de equilibrio espacial e intertemporal.

* Colegio de Postgraduados, México. Correo-e: jsalazar@colpos.mx.

** Universidad Estatal de Nuevo México, Estados Unidos. Correos-e: rskaggs@nmsu.edu, crawford@nmsu.edu. 


\section{Introducción}

Actualmente el Programa de Apoyos Directos al Campo (Procampo) es una política instrumentada por el gobierno de México para apoyar la producción de cultivos básicos, entre ellos el maíz. Este Programa es una contribución directa que el gobierno federal otorga mediante la Secretaría de Agricultura, Ganadería, Desarrollo Rural, Pesca y Alimentación (Sagarpa) para contribuir al ingreso de los productores rurales. El apoyo consiste en la entrega de recursos monetarios por cada hectárea, o fracción sembrada, y registrada en el Programa. El Procampo comenzó a finales de 1993 y respondió a la apertura de la economía nacional y a la necesidad de modernización del campo mexicano (Sagarpa, 2007). Al principio el gobierno anunció que la vigencia del Programa sería de 15 ańos; sin embargo, en el Plan Nacional de Desarrollo 2007-2012 se menciona que continuará hasta el final del sexenio actual mejorando su operación y eficiencia (Presidencia de la República, 2009a).

El Procampo inició apoyando una superficie promedio anual de 13,751 miles de hectáreas en el periodo 1994-1996; sin embargo, en el transcurso de 16 años, dicha superficie se redujo a 11,860 miles de hectáreas en el periodo 2007-2009. Al igual que la superficie, los productores beneficiados por el Programa también disminuyeron: de un padrón de 3,295 miles de productores en 1994, en 2009 sólo se apoyó a 2,121 miles (Presidencia de la República, 2001, 2009b). Del total de la superficie que recibió la ayuda en 2007, 46.6\% se concentró en los estados de Tamaulipas, Zacatecas, Sinaloa, Jalisco, Chihuahua, Guanajuato y Michoacán (Serpro, 2008).

En 2005 se entregaron apoyos por 14,168 millones de pesos a 2.4 millones de productores poseedores de una superficie cultivable de 12 millones de hectáreas, aproximadamente. De estas cantidades, al maíz le correspondieron 6,239 millones de pesos, que beneficiaron a 1.9 millones de productores, con una superficie aproximada de 6.8 millones de toneladas (Sagarpa, 2007). Las cifras anteriores son evidencia de la importancia del maíz para Procampo, pues $44 \%$ de los recursos otorgados fueron para este grano, $56.7 \%$ de la superficie apoyada correspondió al cultivo, y $81.3 \%$ de los agricultores apoyados fueron productores maiceros.

El Programa no sólo ha beneficiado a los productores de maíz. Al principio los cultivos que se eligieron se limitaban a maíz, frijol, trigo, arroz, sorgo, soya, algodón, cártamo y cebada. A partir del ciclo primavera-verano (Pv) 1995 se concretó normativamente la opción para el beneficiario de recibir subsidios por la siembra de cualquier cultivo lícito, lo que permitió estimular la diversificación de la actividad económica y 
propiciar mayor autonomía en la toma de las decisiones del productor (Sagarpa, 2007).

Desde la implementación del Programa, el apoyo otorgado al productor creció en términos nominales: en 1994 fue de 330 pesos/ha en el ciclo otońo-invierno (oI) y de 350 pesos/ha en el ciclo pv; en 2002 fue de 829 pesos/ha en el ciclo oi, y en ese mismo año de 873 pesos/ha en el ciclo PV. A partir de 2003 el pago se realizó de manera indiferenciada: el mismo tanto durante el ciclo or como el que se otorgó a los productores de riego del ciclo pv, y la cuota preferencial dada a los productores de temporal del ciclo Pv; en el periodo 2005-2009 la cuota normal pagada en ambos ciclos se mantuvo constante en 963 pesos/ton, al igual que la cuota preferencial, la cual se ubicó en 1,160 pesos/ton (Presidencia de la República, 2001, 2009b).

El estancamiento del pago Procampo en términos nominales originó una disminución del mismo en términos reales; durante el periodo 1994/1996-2007/2009 la cuota normal en términos reales pagada en los ciclos or y PV disminuyó a un ritmo anual de 2.6 y $3.2 \%$, en tanto que en el periodo la disminución fue de 28.8 y $34.4 \%$. La misma tendencia presentó la cuota preferencial, la cual disminuyó a un rimo anual de $2.8 \%$, durante el periodo 2003/2005-2007/2009 (Presidencia de la República, 2001, 2009b).

Al igual que la cuota, los recursos totales otorgados por el programa crecieron en términos nominales, aunque no al mismo ritmo de la inflación. En el primer año, los recursos anuales promedio canalizados anualmente fueron 4,848 millones de pesos en el periodo 2003-2005, y para 2007-2009 éstos se ubicaron en 14,209 millones de pesos, lo anterior representó un crecimiento medio anual de $7.1 \%$. Debido a que no aumentaron al mismo ritmo de la inflación, los recursos otorgados por Procampo disminuyeron en términos reales; durante el periodo 1994/1996-2007/2009 los recursos totales bajaron a un ritmo anual de 2.6\% (Presidencia de la República, 2001, 2009b).

A más de 16 años de vigencia existe una gran cantidad de estudios sobre Procampo, con diferentes enfoques y distintos objetivos. Dichos estudios se han enfocado a analizar los efectos del programa en variables como el ingreso de los productores, la pobreza, la producción, etc. Una clasificación de los diferentes estudios que podría ser útil para la principal conclusión derivada de este trabajo es la siguiente: $a$ ) aquellos estudios que se centran en los aspectos positivos del programa, y $b$ ) los que puntualizan los efectos negativos. Las dos perspectivas se pueden analizar en el marco de los objetivos que se propuso alcanzar Procampo.

Brindar apoyo a más de tres millones de productores y compensar los subsidios otorgados en otros países fueron dos objetivos del Programa, 
quizá ésta sea la razón de por qué el ingreso es una de las variables más estudiadas. Existe evidencia empírica de que las transferencias de Procampo han tenido un efecto multiplicador considerable en el ingreso de los hogares, y que el pago directo ha generado aumentos en el ingreso para los beneficiarios más pobres (Sadoulet et al., 2001; Tangerman, 2006).

Algunas evaluaciones indican que Procampo cumplió con el objetivo de aumentar los ingresos de los productores, no sólo por el subsidio directo que otorga, sino por la vía de generar un mayor valor de la producción para los beneficiarios, en comparación con las unidades de producción que no lo recibieron. Dicho incremento en el valor de la producción no se derivó de mayores superficies, sino necesariamente de un mayor rendimiento por hectárea, de mejores condiciones para la comercialización, de más inversiones por parte de los productores beneficiados y de mejores condiciones de acceso al financiamiento para la producción (GEA, 2005).

Otro de los objetivos de Procampo fue lograr que los consumidores nacionales tengan acceso a alimentos a más bajos precios. Sobre este objetivo se ha concluido que el costo de la canasta de productos básicos (maíz, frijol, arroz, trigo y sorgo) tendió a disminuir a lo largo del tiempo. Esta tendencia se acentúo a partir de 1994, fecha en que comenzó el Programa y el TLCAN. El costo de dicha canasta fue menor en el periodo Procampo que en el de precios de garantía. La política de pagos directos resultó en un beneficio para los compradores de dichos productos a nivel productor. Al retirar la política de precios de garantía el costo de la canasta de productos básicos disminuyó 56.3\% durante el periodo Procampo (Hernández y Martínez, 2009).

Aunque no se plasmó como objetivo, los efectos en la migración han sido tema principal de otros estudios. Se ha concluido que el efecto de Procampo en la migración, tanto temporal como permanente, ha sido negativo (González y Wodon, 2005).

Los efectos en la producción de maíz, el cultivo más favorecido por el Programa, también han sido tema de estudio. Análisis econométricos concluyen que aunque la relación entre el pago de Procampo y la producción de maíz es inelástica en las distintas zonas productoras del país, el programa es positivo debido a que más de $15 \%$ de la producción promedio observada en el periodo 1994-1996 se debe a su existencia (García, 2001). El mismo autor concluye que si bien la desaparición de Procampo significaría un ahorro considerable de recursos, pues la producción de maíz disminuiría en más de dos millones de toneladas, y con los recursos liberados se podrían importar más de cuatro millones de toneladas, el programa debe permanecer porque brinda apoyo directo a más de tres millones de productores. 
Otros estudios indican que el efecto de Procampo se ha dado mediante el mejoramiento de la tierra y rendimientos por hectárea, ya que 51, 75 y $100 \%$ del apoyo recibido por los productores pequeños, medianos y grandes, respectivamente, se invertió en actividades agrícolas (García, 2004); dicha asignación presupone una influencia positiva del Programa en el desempeño productivo del sector.

Otros estudios han puntualizado sus efectos negativos, y quizá el aspecto más criticado es la distribución inadecuada de los recursos entre los productores. Se ha señalado que los hogares que reciben la ayuda tienen una distribución que se concentra en los deciles de más altos ingresos. En 1996 los tres primeros deciles concentraron casi 10\% del ingreso, mientras que los tres últimos concentraron poco más de 60\%. En 1998 los tres primeros deciles concentraron $10.7 \%$ del ingreso y los tres últimos $57.3 \%$ (Herrera, 2002). El mismo autor sostiene que si uno de los efectos perceptibles del Programa es la concentración del ingreso, es posible pensar que los objetivos de apoyar a quienes producen para el autoconsumo y mejorar el nivel de ingreso, no se hayan cumplido. Sobre este mismo tema, Scott (2007) informa que $2.6 \%$ de productores en el decil nacional más rico, captaron $23 \%$ de las transferencias.

Otro aspecto negativo que se señala es que Procampo no ha inducido transición a cultivos de mayor rentabilidad. Después de una década, cerca de la mitad de los beneficiarios (pequeños y grandes productores por igual) dicen ignorar que pueden plantar cualquier cultivo sin perder los beneficios. Sólo 5.8\% informa que cambió de cultivos (Scott, 2007). Esto evidencia que el objetivo de fomentar la reconversión productiva de aquellas superficies en las que sea posible establecer actividades con mayor rentabilidad, no se ha cumplido de manera satisfactoria.

Las evaluaciones del programa seńalan, precisamente, que los retos se centran en dos aspectos: la conversión de cultivos y la distribución del ingreso. Una parte importante de los beneficiarios no está enterada de las reglas del Programa en lo que se refiere a la conversión productiva: $47.7 \%$ de las unidades de producción manifestó no saber que se puede sembrar cualquier cultivo sin perder el apoyo, y este patrón se presenta en todos los estratos de productores (GEA, 2005).

Aparte de los retos que enfrenta, actualmente se reconoce que existen problemas que limitan los objetivos de Procampo; entre los más importantes destacan los siguientes: a) el Programa benefició más a los productores que tienen más tierra; $b$ ) muchos de los productores recibieron el subsidio después de la siembra; c) la cuota de Procampo disminuyó en términos reales por efecto de la inflación, y d) un buen porcentaje de los productores no destinó la cuota a actividades agrícolas. Estos problemas han sido analizados por funcionarios y líderes de organizaciones agrícolas, 
quienes plantean propuestas para mejorar el funcionamiento del Programa en un futuro (Davis, 2007; Rosenzweig, 2007; Labarthe, 2007; Kondo, 2007; Martínez, 2007).

La tendencia de las principales variables del mercado de maíz en México indica que el Programa ha favorecido tanto la producción como el consumo. Durante el periodo 1991-1993, la producción anual promedio de maíz fue de 15.3 millones de toneladas, en tanto que durante 2007-2009 ascendió a 24.6 millones, lo cual representó un crecimiento medio anual de $2.8 \%$, y $61.2 \%$ en el periodo. Las fuentes de crecimiento indican que la subida de la producción provino sobre todo del aumento en los rendimientos, los cuales crecieron a un ritmo anual de $2.7 \%$, en tanto que la superficie contribuyó creciendo a un ritmo anual de $0.1 \%$ en el periodo señalado (Presidencia de la República, 2001, 2009b).

A pesar de la alta producción de maíz alcanzada en el periodo 19942009, las importaciones se caracterizaron por un aumento constante. En 1994 las compras externas del grano se ubicaron en 2.7 millones de toneladas, alcanzando una cifra récord en 2008, año en que se ubicaron en 9.1 millones de toneladas (Presidencia de la República, 2009b). El aumento de las importaciones, a un ritmo anual de $8.1 \%$ en el periodo 1991/1993-2007/2209, es evidencia de que la producción de maíz, al igual que otros productos básicos, presenta el problema de falta de competitividad en el escenario actual de apertura comercial. Los productores del grano no ganaron los nuevos mercados regionales y permitieron que los extranjeros se apoderaran de éstos (García et al., 2006).

Como consecuencia del aumento de la producción y de las importaciones, el consumo anual promedio experimentó un crecimiento importante: pasó de 17.5 millones de toneladas al año en 1991-1993 a 33.9 millones en el periodo 2007-2009. Cabe destacar que la parte más dinámica en el crecimiento experimentado por el consumo nacional debe atribuirse al maíz consumido por el ganado. Dicho aumento en la demanda fue consecuencia de la disminución del precio medio del maíz en términos rurales, el cual experimentó un descenso anual de 3.7\% en el periodo 1994/1996-2007/2008 (sIAP-Sagarpa, 2009a).

Los estudios que han analizado los efectos de Procampo se centraron de manera separada en las distintas variables afectadas (producción e ingresos, principalmente), y no consideraron los cambios en el bienestar de la sociedad. Además de medir los efectos en el ingreso y la producción, una evaluación de los efectos del Programa debería incluir el efecto en el empleo de mano de obra, y el cambio en el excedente al consumidor, excedente al productor $\mathrm{y}$ valor de las importaciones.

Considerando la importancia que tiene Procampo como principal instrumento de apoyo a la producción de maíz, este trabajo tiene como 
objetivo medir sus efectos en la producción, el consumo, las importaciones, el empleo y bienestar de la sociedad. Dicha medición pretende dimensionar los beneficios de la política frente al gasto gubernamental que su implementación significa. Debido a que los subsidios otorgados por el lado de la oferta elevan la producción y el excedente al productor, y permiten ahorrar divisas por las menores importaciones, se espera que los beneficios derivados de la implementación del Programa sean mayores a los gastos del gobierno.

Este trabajo está organizado en los siguientes temas: el primero presenta los antecedentes del problema formal y los objetivos de la investigación; el segundo aborda el modelo que se utilizó para alcanzar los objetivos, así como las fuentes de información; el tercero es una discusión de los resultados; finalmente se exponen las conclusiones.

\section{Metodología}

Para alcanzar el objetivo se usó un modelo de equilibrio espacial e intertemporal. Se prefirió este tipo de modelos porque permite, por un lado, replicar el funcionamiento del mercado de forma completa porque usa información desagregada espacialmente sobre consumo, producción, precios al productor y consumidor, importaciones, flujos comerciales, costos de transporte y otros costos de comercialización y, por otro, determinar los efectos de cambios de políticas (subsidios, aranceles, tasa de cambio, etc.) sobre las variables endógenas del sistema. Su principal desventaja es la gran cantidad de información que se requiere, ya que necesita un considerable grado de desagregación en la información utilizada a nivel espacial y temporal, a diferencia de otros modelos, como los de ecuaciones simultáneas que utilizan datos nacionales y anuales, y capturan de forma reducida los efectos de cambios exógenos sobre las principales variables del mercado (oferta, demanda, importaciones y precios).

Entre los estudios que han usado modelos de equilibrio espacial destacan los siguientes: Kawaguchi et al. (1997) analizaron los flujos comerciales de la leche en Japón suponiendo diferentes estructuras de mercado, y Cramer et al. (1993) estudiaron los efectos de la liberación comercial en el mercado internacional del arroz. Boyd et al. (1993) investigaron el efecto de la liberación comercial entre Estados Unidos, México y Canadá sobre la producción y flujos comerciales de madera. Por su parte, Bivings (1997) estudió el efecto de la liberación del mercado de sorgo en la producción, demanda e importaciones del grano en México. García y Williams (2004) analizaron cómo la política comercial, el tipo de cambio y los costos de transporte afectaron el mercado de maíz en México. García et al. (2005) midieron el efecto de la liberación comercial acordada en el 
TLCAN sobre las exportaciones de tomate rojo en fresco de México a Estados Unidos. También usando un modelo de equilibrio espacial e intertemporal, Rebollar et al. (2006) determinaron la demanda óptima de almacenamiento para determinar regiones con excesos y déficits de almacenes. Torres y García (2008) usaron un modelo de equilibrio espacial para determinar la estructura que rige el funcionamiento del mercado de frijol en México.

El modelo usado en esta investigación consideró los diferentes agentes que participan en el mercado de maíz: productores, consumidores, importadores, intermediarios y almacenes. La formulación del modelo se basó en Takayama y Judge (1971) y Bivings (1997), y consideró el consumo (animal, humano e industrial), la producción e importaciones (de maíz blanco y amarillo) y dos formas de transporte (camión y ferrocarril). Se usó un modelo de este tipo porque permite ver cómo los cambios en el pago de Procampo tienen efecto en las variables del mercado. Suponiendo $i(i=1,2 \ldots I=10)$ regiones productoras de maíz blanco, $s(s=1,2 \ldots$ $S=10)$ regiones productoras de maíz amarillo, $j(j=1,2 \ldots J=10)$ regiones de consumo animal, $d(a=1,2,3 \ldots D=10)$ regiones de consumo humano, $f(f=1,2,3 \ldots F=10)$ regiones de consumo industrial, y $t(t=1,2 \ldots T$ = 12) periodos, las funciones inversas de oferta y demanda de maíz están dadas por las siguientes funciones lineales:

$$
\begin{aligned}
& p_{i t}=v_{i t}+\kappa_{i t} p c_{i t}+\eta_{i t} x_{i t} \\
& p_{s t}=v_{s t}+\kappa_{s t} p c_{s t}+\eta_{s t} x_{s t} \\
& p_{j t}=\lambda_{j t}+\omega_{j t} y_{j t} \\
& p_{d t}=\lambda_{d t}+\omega_{d t} y_{d t} \\
& p_{f t}=\lambda_{f t}+\omega_{f t} y_{f t}
\end{aligned}
$$

donde $x_{i t}$ y $x_{s t}$ son la cantidades ofrecidas de maíz blanco y amarillo, $p_{i t} \mathrm{y}$ $p_{s t}$ son los precios al productor de maíz blanco y amarillo, $y_{j t} y_{d t}$ y $y_{f t}$ son los consumos animal, humano e industrial de maíz, $p_{j l}, p_{d t}$ y $p_{f t}$ son los precios al consumidor, $p c_{i t} y p c_{s t}$ son el pago de Procampo al maíz blanco y amarillo. La función de oferta supone que la cantidad ofrecida depende del precio y del subsidio otorgado mediante el Programa.

Suponiendo $m(m=1,2 \ldots M=11)$ fronteras y puertos de entradas de importaciones de maíz blanco, $n(n=1,2 \ldots N=11)$ fronteras y puertos de entrada de importaciones de maíz amarillo, y $r(r=1, . . T=2)$ formas de transporte, el modelo de programación se puede formular de la siguiente manera: 


$$
\begin{aligned}
& \operatorname{MaxVSN}=\sum_{t=1}^{T} \pi^{t-1} \sum_{j=1}^{J}\left[\lambda_{j t} y_{j t}+\frac{1}{2} \omega_{j t} y_{j t}^{2}\right]+\sum_{t=1}^{T} \pi^{t-1} \sum_{d=1}^{D}\left[\lambda_{d t} y_{d t}+\frac{1}{2} \omega_{d t} y_{d t}^{2}\right] \\
& +\sum_{t=1}^{T} \pi^{t-1} \sum_{f=1}^{F}\left[\lambda_{f} y_{f t}+\frac{1}{2} \omega_{f} y_{f t}^{2}\right] \sum_{t=1}^{T} \pi^{t-1} \sum_{i=1}^{I}\left[\underline{L}_{i t} x_{i t}+\kappa_{i t} p c_{i t}+\frac{1}{2} \eta_{i t} x_{i t}^{2}\right] \\
& -\sum_{t=1}^{T} \pi^{t-1} \sum_{s=1}^{S}\left[\underline{v}_{s t} x_{s t}+\kappa_{s t} p c_{s t}+\frac{1}{2} \eta_{s t} x_{s t}^{2}\right]-\sum_{t=1}^{T} \pi^{t-1} \sum_{m=1}^{M}\left[p_{m t} x_{m t}\right]-\sum_{t=1}^{T} \pi^{t-1} \sum_{n=1}^{N}\left[p_{n t} x_{n t}\right] \\
& -\sum_{i=1}^{T} \pi^{t-1} \sum_{i=1}^{T} \sum_{j=1}^{J} \sum_{r=1}^{R}\left[p_{i j r r t} x_{i j r t}\right]-\sum_{i=1}^{T} \pi^{t-1} \sum_{i=1}^{I} \sum_{d=1}^{D} \sum_{r=1}^{R}\left[p_{i d r t} x_{i d r t}\right]-\sum_{i=1}^{T} \pi^{t-1} \sum_{s=1}^{S} \sum_{j=1}^{J} \sum_{r=1}^{R}\left[p_{s j r} x_{s j r t}\right] \\
& -\sum_{t=1}^{T} \pi^{t-1} \sum_{s=1}^{S} \sum_{j=1}^{F} \sum_{r=1}^{R}\left[p_{s f r} x_{s f r t}\right]-\sum_{t=1}^{T} \pi^{t-1} \sum_{m=1}^{M} \sum_{j=1}^{S} \sum_{r=1}^{R}\left[p_{m j r} x_{m j r t}\right]-\sum_{t=1}^{T} \pi^{t-1} \sum_{m=1}^{M} \sum_{d=1}^{D} \sum_{r=1}^{R}\left[p_{m d r t} x_{m d r t}\right] \\
& -\sum_{t=1}^{T} \pi^{t-1} \sum_{n=1}^{N} \sum_{j=1}^{J} \sum_{r=1}^{R}\left[p_{n j r} x_{n j r t}\right] \sum_{t=1}^{T} \pi^{t-1} \sum_{n=1}^{N} \sum_{j=1}^{F} \sum_{r=1}^{R}\left[p_{n f r} x_{n f r t}\right] \sum_{t=1}^{T} \pi^{t-1} \sum_{i=1}^{I}\left[p_{i t, t+1} x_{i t, t+1}\right] \\
& -\sum_{t=1}^{T} \pi^{t-1} \sum_{s=1}^{S}\left[p_{s t, t+1} x_{s t, t+1}\right]-\sum_{t=1}^{T} \pi^{t-1} \sum_{m=1}^{M}\left[p_{m t, t+1} x_{m t, t+1}\right]-\sum_{t=1}^{T} \pi^{t-1} \sum_{n=1}^{N}\left[p_{n t, t+1} x_{n t, t+1}\right]
\end{aligned}
$$

Sujeto a las siguientes restricciones:

$$
\begin{aligned}
& x_{i t}+x_{i t-1, t}-x_{i t, t+1} \geq \sum_{j=1}^{J} \sum_{r=1}^{R}\left[x_{i j r t}\right]+\sum_{d=1}^{D} \sum_{r=1}^{R}\left[x_{i d r t}\right] \\
& x_{s t}+x_{s t-1, t}-x_{s t, t+1} \geq \sum_{j=1}^{J} \sum_{R}^{R}\left[x_{s j r t}\right]+\sum_{f=1}^{T} \sum_{r=1}^{R}\left[x_{s f r t}\right] \\
& x_{m t}+x_{m t-1, t}-x_{m t, t+1} \geq \sum_{j=1}^{J} \sum_{r=1}^{R}\left[x_{m j r t}\right]+\sum_{d=1}^{D} \sum_{r=1}^{R}\left[x_{m d r t}\right] \\
& x_{n t}+x_{n t-1, t}-x_{n t, t+1} \geq \sum_{j=1}^{J} \sum_{r=1}^{R}\left[x_{n j r t}\right]+\sum_{f=1}^{F} \sum_{r=1}^{R}\left[x_{n f r t}\right] \\
& \sum_{i=1}^{I} \sum_{r=1}^{R}\left[x_{i j r t}\right]+\sum_{s=1}^{S} \sum_{r=1}^{R}\left[x_{s j r t}\right]+\sum_{m=1}^{M} \sum_{r=1}^{R}\left[x_{m j r t}\right]+\sum_{n=1}^{N} \sum_{r=1}^{R}\left[x_{n j r t}\right] \geq y_{j t} \\
& \sum_{i=1}^{I} \sum_{r=1}^{R}\left[x_{i d r t}\right]+\sum_{m=1}^{M} \sum_{r=1}^{R}\left[x_{m d r t}\right] \geq y_{d t} \\
& \sum_{s=1}^{S} \sum_{r=1}^{R}\left[x_{s f r t}\right]+\sum_{n=1}^{N} \sum_{r=1}^{R}\left[x_{n f r t}\right] \geq y_{f t} \\
& x_{i 12,13}=x_{i 0,1} \\
& x_{s 2,13}=x_{s 0,1} \\
& x_{t}=\sum_{m=1}^{M} x_{m t} \\
& \sum_{t=1}^{T} \sum_{m=9}^{M}\left[x_{m t}\right]+\sum_{t=1}^{T} \sum_{n=9}^{N}\left[x_{n t}\right]=\delta\left[\sum_{t=1}^{T} \sum_{m=1}^{M}\left[x_{m t}\right]+\sum_{t=1}^{T} \sum_{n=1}^{N}\left[x_{n t}\right]\right] \\
& y_{j t}, y_{d t}, y_{f o} x_{i t}, x_{s t}, x_{m t}, x_{n t}, \ldots \ldots \ldots \ldots . . . x_{i t, t+1}, x_{s t, t+1}, x_{m t, t+1}, x_{n t, t+1} \geq 0
\end{aligned}
$$


donde para el mes $t: \pi^{t-1}=(1 / 1+i)^{t-1}$ es el factor de descuento con $i_{t}$ igual a la tasa de inflación; $\lambda_{j t}, \lambda_{d t}$ y $\lambda_{f t}$, son las ordenadas al origen de las funciones de demanda de maíz en las regiones $j, d$ y $f ; y_{j t}, y_{d t}$ y $y_{f t}$ son las cantidades de maíz consumidos por los sectores animal, humano e industrial en las regiones $j, d y f ; \omega_{j t}, \omega_{d t}$ y $\omega_{f t}$ son las pendientes de las funciones de demanda en las regiones $j, d y f ; v_{i t}$ y $v_{s t}$ son las ordenadas al origen de funciones de oferta de maíz en las regiones $i$ y $s ; x_{i t}$ y $x_{s t}$ son las cantidades producidas de maíz blanco y amarillo en las regiones $i$ y $s ; \eta_{i t}$ y $\eta_{s t}$ son las pendientes de las funciones de oferta en las regiones $i$ y $s ; p_{m t}$ y $p_{n t}$ son los precios internacionales de maíz blanco y amarillo importado a través del puerto/frontera $m$ y $n ; x_{m t}$ y $x_{n t}$ son las cantidades de maíz blanco y amarillo importadas mediante los puertos/fronteras $m$ y $n$; $p_{i j r t}$ y $x_{i j r t}$ son los costos de transporte y cantidades enviadas de maíz blanco, usando $r$, de $i$ a $j ; p_{i d r t}$ y $x_{i d r t}$ son los costos de transporte y cantidades enviadas de maíz blanco, usando $r$, de $i$ a $d ; p_{s j r t}$ y $x_{\text {sjrt }}$ son los costos de transporte y cantidades enviadas de maíz amarillo, usando $r$, de $s$ a $j$; $p_{\text {sfit }}$ y $x_{s f i t}$ son los costos de transporte y cantidad enviada de maíz amarillo, usando $r$, de $s$ a $f ; p_{m j r t}$ y $x_{\text {mirt }}$ son los costos de transporte y cantidad enviada de maíz blanco, usando $r$, de $m$ a $j ; p_{m d r t}$ y $x_{m d r t}$ son los costos de transporte y cantidad enviada de maíz blanco, usando $r$, de $m$ a $d ; p_{\text {nirt }} \mathrm{y}$ $x_{n j t}$ son los costos de transporte y cantidad enviada de maíz amarillo, usando $r$, de $n$ a $j ; p_{n f i t}$ y $x_{n f i t}$ son los costos de transporte y cantidad enviada de maíz amarillo, usando $r$, de $n$ a $f ; p_{i t, t+1}$ y $p_{s t, t+1}$ son los costos de almacenamiento por unidad de maíz blanco y amarillo en las regiones $i$ y $s$ del mes $t$ al mes $t+1 ; x_{i t, t+1}$ y $x_{s, t+1}$ son las cantidades de maíz blanco y amarillo almacenadas en las regiones $i$ y del mes $t$ al mes $t+1 ; p_{m t, t+1}$ y $p_{n, t+1}$ son los costos de almacenamiento por unidad de maíz blanco y amarillo almacenados en los puertos $m$ y $n$ del mes $t$ al mes $t+1 ; x_{m t, t+1}$ y $x_{n t, t+1}$ son las cantidades de maíz blanco y amarillo almacenadas en los puertos $m$ y $n$ del mes $t$ al mes $t+1$.

La función objetivo del modelo maximiza el valor social neto (vsN), el cual es igual a la sumatoria de las áreas bajo las curvas de demanda, menos el área bajo las curvas de oferta, menos el valor de las importaciones y menos los costos de transporte y almacenamiento. Las restricciones 7 y 8 (9 y 10) indican cómo se distribuye la producción (importaciones) de maíz blanco y amarillo. Las restricciones 11, 12 y 13 establecen cómo se abastece el consumo animal, humano e industrial. Las restricciones 14 y 15 indican que los inventarios finales en la regiones $i$ y $s$ son iguales a los inventarios iniciales. Para incorporar escasez de maíz blanco en Estados Unidos, se estableció la restricción 16, que indica que las importaciones que entran a través de puertos/fronteras en un mes deben ser iguales a los datos observados en el año 2005/2007. Para reflejar la capa- 
cidad de la infraestructura de transporte y almacenamiento que existe en los puertos del Golfo se estableció la restricción 17, que indica que las importaciones de maíz blanco y amarillo que entraron por Nuevo Laredo, Piedras Negras y Ciudad Juárez deben representar $60.1 \%(\delta)$ de las importaciones totales, correspondiente a la participación porcentual durante 2005/2007. Finalmente, la restricción 18 establece las condiciones de no negatividad del modelo.

Una vez que se obtuvo la solución del modelo, el empleo anual de mano de obra $\left(e_{a}\right)$ se calculó de acuerdo con la ecuación 19.

$$
e_{a}=\frac{x_{i}}{r_{i}} \times e_{i}
$$

donde $x_{i}$ es la producción anual de maíz en la región $i$ (igual a $\left.\sum_{i=1}^{T} x_{i t}+\sum_{t=1}^{T} x_{s}\right), r_{i}$ son los rendimientos de maíz en la región $i$, y $e_{i}$ son los requerimientos de mano de obra por hectárea en la región $i$.

El modelo incluyó 10 regiones productoras de maíz blanco: Chiapas, Jalisco, Centro, Guanajuato-Michoacán, Sinaloa, Golfo, GuerreroOaxaca, Norte, Noreste y Península; ${ }^{1}$ y las mismas regiones se consideraron para la producción de maíz amarillo y para consumo animal, humano e industrial. Se consideraron 11 puertos/fronteras de entrada de importaciones: Mexicali, Nogales, Ciudad Juárez, Piedras Negras, Nuevo Laredo, Tampico, Veracruz, Progreso, Guaymas, Mazatlán y Manzanillo.

Para determinar los efectos de la implementación del Procampo, o en su caso el aumento del apoyo, primero se estimó y validó el modelo para el año promedio 2005/2007. Para cada región, el promedio de tres ciclos anuales de consumo se usaron iniciando en octubre de 2004 y finalizando en septiembre de 2007; de octubre a diciembre todos los valores son datos promedio correspondientes a 2004, 2005 y 2006, y de enero a septiembre todos los valores son promedio de 2005, 2006 y 2007. El año que va de octubre de un año a septiembre del siguiente se definió como año promedio 2005/2007.

Se obtuvo una gran cantidad de soluciones para validar el modelo, y el procedimiento usado para realizar la validación consistió en comparar los valores observados de la producción de maíz, el consumo y los precios

${ }^{1}$ Jalisco incluye Aguascalientes y Colima; el Centro a Estado de México, Morelos, Distrito Federal, Querétaro, Hidalgo, Puebla y Tlaxcala; Sinaloa incluye Baja California, Baja California Sur, Nayarit y Sonora; el Golfo a Veracruz y Tabasco; el Norte incluye Chihuahua, Coahuila, Durango, San Luis Potosí y Zacatecas; el Noreste a Nuevo León y Tamaulipas; la Península incluye Yucatán, Campeche y Quintana Roo. 
al consumidor en el año promedio 2005/2007, con los valores obtenidos por el modelo base. Una vez que la mayoría de las diferencias (en porcentaje) entre los valores observados y los valores estimados por el modelo fueron menores de $10 \%$, se seleccionó el modelo base. En el proceso de validación se probaron diferentes coeficientes de elasticidad y distintos niveles de precios al productor y consumidor, y se seleccionaron aquéllos que permitieron replicar el valor de las variables endógenas del sistema. Las fuentes de información de donde provinieron las elasticidades y precios se mencionan en la sección de datos. Es importante mencionar que hubiera sido deseable el uso de elasticidades a nivel regional, sin embargo, éstas no se usaron por las siguientes razones: a) no hay estimaciones actualizadas de dichos coeficientes, y $b$ ) las elasticidades nacionales permitieron obtener un modelo base que replica de forma adecuada las principales variables del mercado de maíz en México.

El modelo base representa la situación en la que existe Procampo. Una vez que se obtuvo el modelo base se realizaron los siguientes escenarios: a) disminución del pago Procampo en 100\% (desaparición del programa); y b) aumento del pago en $50 \%$. Usando las cantidades ofrecidas, demandas e importadas, y los precios, se calcularon los excedentes al productor, al consumidor y el valor de las importaciones en los diferentes escenarios. Para el cálculo del excedente al productor, sólo se consideró el área bajo la curva de oferta positiva.

Las funciones de oferta y demanda se calcularon usando cantidades producidas y consumidas de maíz, precios al productor y consumidor, elasticidades precio de la oferta y demanda, elasticidades que miden la relación entre la producción y el pago de Procampo y el apoyo otorgado a través del mismo. Las elasticidades precio de la demanda para el consumo animal y humano se tomaron del Food and Agricultural Policy Research Institute (FAPRI, 2009). El FAPRI reporta elasticidades precio para la demanda animal y humana en el ámbito nacional y éstas se usaron en el modelo para todas las regiones y periodos. Se supuso que la elasticidad precio para la demanda industrial fue la misma que la que reporta el FAPRI para consumo humano. La elasticidad precio de la oferta de maíz también provino de FAPRI (2009), que informa coeficientes para todo el país. La elasticidad pago de Procampo de la producción de maíz provino de García (2001). La producción de maíz en México por región y mes se obtuvo del Sistema de Información Agroalimentaria y Pesquera de la Sagarpa (2009b) y el pago del Programa provino de la Presidencia de la República (2009b).

El consumo de maíz por región y mes se calculó de la siguiente forma: a) primero se estimó el consumo nacional aparente (CNA) sumando la producción nacional de maíz blanco y amarillo más las importaciones de 
éstos, menos las exportaciones por año, y posteriormente se desagregó según su uso en consumo animal, humano, industrial, semilla para siembra y mermas; b) el consumo de semilla para siembra se obtuvo multiplicando la superficie sembrada en hectáreas (ha) por la densidad de siembra $(\mathrm{kg} / \mathrm{ha})$, y para obtener las mermas poscosecha se asumió que $4.14 \% \mathrm{de}$ la producción de maíz se perdió en el proceso de comercialización; c) el consumo anual animal en todo el país se ponderó por la participación de cada región en el consumo nacional de granos para obtener el consumo de maíz como forraje en el ámbito regional; $d$ ) de manera similar, el consumo humano (industrial) anual en todo el país se ponderó por la participación de cada región en la industria del nixtamal y molienda (almidones) para obtener los consumos alimentario e industrial en el ámbito regional; $e$ ) se asumió que el consumo animal, humano e industrial se distribuye de manera igual durante los 12 meses del año. La información usada para calcular el consumo se obtuvo de siap-Sagarpa (2009b), de United States International Trade Comission (Usitc, 2009), de la Sagarpa (2007) y del Instituto Nacional de Estadística Geografía e Informática (INEGI, 1995).

Los precios regionales pagados por los consumidores y recibidos por los productores se calcularon usando el precio internacional de maíz en puntos de entrada de las importaciones en México. Se sumaron los costos de transporte de cada puerto y frontera de entrada de las importaciones al precio internacional para obtener precios al consumidor. Se asumió que los precios al productor en cada región fueron iguales al precio al consumidor menos un margen de comercialización para transportar el grano de la finca a los puntos de consumo. Los márgenes de comercialización en cada región se estimaron usando la diferencia entre el precio promedio al mayoreo del maíz en las centrales de abasto y los precio promedio recibidos por los productores. Los datos para obtener el precio promedio al productor provinieron de SIAP-Sagarpa (2009a) y los precios promedio al mayoreo se obtuvieron del Sistema Nacional de Información e Integración de Mercados (SNIIM, 2009).

Los precios internacionales del maíz amarillo en las fronteras mexicanas se calcularon con base en los precios de maíz en Iowa más los costos de transporte de Des Moines, Iowa, a las fronteras de Eagle Pass y Laredo, Texas. El precio internacional de maíz en puertos mexicanos del Golfo y Pacífico se estimaron usando los precios de maíz en el puerto de Nueva Orleans, más los costos de seguro y flete marítimo de Nueva Orleans a los puertos mexicanos. El precio internacional de maíz se calculó tomando en cuenta la tasa de cambio, el seguro y flete marítimo, el costo financiero internacional y los gastos portuarios o de cruce. De acuerdo con la Sagarpa (2007), se consideró que el precio internacional del maíz blanco 
fue igual al precio del maíz amarillo más un sobreprecio de 13\%. Los precios del maíz en Iowa y Nueva Orleans se obtuvieron del Agricultural Marketing Service (AMS-USDA, 2009), los costos de transporte de Des Moines a Eagle Pass y Laredo se obtuvieron del AMs-usDA (2009).

Los costos de entrada y de cruce se tomaron de Apoyos y Servicios a la Comercialización Agropecuaria (Aserca). Los datos sobre seguro y flete marítimo se obtuvieron del AMs-USDA (2009). La tasa de cambio se tomó del INEGI (2008a) y la tasa libor para calcular el costo financiero internacional se obtuvo del INEGI (2008c). Los requerimientos de mano de obra por hectárea provinieron de la Sagarpa (2007).

Los costos de transporte por ferrocarril de las regiones productoras y puertos/fronteras de entrada de las importaciones a los puntos de consumo se estimaron usando una matriz de distancias y una tarifa promedio que incluye un factor fijo y un factor variable. Los datos de la tarifa provinieron de la Secretaría de Comunicaciones y Transporte (sCT, 2009). Las ciudades tomadas como referencia para calcular los costos de transporte fueron Tapachula, Guadalajara, ciudad de México, Morelia, Culiacán, Jalapa, Oaxaca, Chihuahua, Monterrey y Mérida. En forma similar, los costos de transporte por camión de las zonas productoras y puertos/ fronteras de entrada a los centros de consumo se estimaron usando una matriz de distancias con datos provenientes de la Dirección General de Tarifas, Transporte Ferroviario y Multimodal (DGTTFM, 2000). Los datos de dicha Dirección se actualizaron a los años 2005-2007 usando el índice nacional de precios al consumidor del sector transporte. Las ciudades tomadas como punto de referencia fueron Tapachula, Guadalajara, ciudad de México, Morelia, Culiacán, Jalapa, Oaxaca, Chihuahua, Monterrey y Mérida. Finalmente, los costos de almacenamiento de maíz en las zonas productoras y puertos de entrada de las importaciones, que incluyen maniobras de entrada y salida, se estimaron con base en información de la Sagarpa (2002) y del INEGI (2008b).

\section{Resultados y análisis}

El modelo se validó con el consumo regional, la producción, los precios al consumidor y las importaciones totales. En el cuadro 1 se presentan los resultados que se obtienen con el modelo base y se comparan con los valores observados en el año 2005/2007. Excepto en pocos casos, la solución del modelo fue muy cerrada a los niveles observados en el año de análisis. En el ámbito nacional el modelo sobreestima el consumo y la producción en $+1.5 \mathrm{y}+2.1 \%$, respectivamente, de manera similar, el modelo base subestima las importaciones de maíz y los precios al consumidor en -0.03 y $-10.1 \%$, respectivamente. En el ámbito regional, el 
modelo sobreestima la producción de maíz blanco en el Noreste en $+9.6 \%$, sin embargo, el efecto sobre la producción total de maíz blanco es pequeña. De manera similar, el modelo subestima el precio al consumidor en las regiones de Chiapas, Guanajuato-Michoacán y Oaxaca-Guerrero en $-18.1,-18.4$ y -17.7\%; sin embargo, en estas regiones el consumo de maíz es pequeño.

En el año promedio 2005/2007, el consumo y la producción total de maíz ascendieron a 28'606,000 y 21'390,000 toneladas métricas, respectivamente, lo cual determinó importaciones por 7’216,000 toneladas (cuadro 1). Las altas importaciones son evidencia de la pérdida de autosuficiencia alimentaria y la dependencia del mercado estadounidense; en el año de análisis una cuarta parte del consumo se abasteció con maíz proveniente del exterior, y sólo tres cuartas partes se hicieron con producción doméstica.

Conviene destacar que el consumo, la producción y las importaciones del cuadro 1 consideran la existencia del subsidio otorgado por Procampo, esto significa que sin el Programa la producción sería menor y las importaciones mayores. En el cuadro 2 se presentan los resultados de la solución del modelo de programación en el escenario que considera la inexistencia del Procampo. La eliminación de éste disminuiría la producción y el empleo de mano de obra en 3'175,000 toneladas y 12'531,000 jornales, en relación con los niveles observados en 2005/2007, lo cual indica que el Programa tuvo un efecto positivo sobre ambas variables. De manera similar, la desaparición del Programa disminuiría el consumo en 250,000 toneladas, en relación con el nivel observado con la existencia del mismo.

Estos resultados son similares a los que encontró García (2001), quien usó un modelo de ecuaciones simultáneas para determinar los efectos del Programa en el mercado de maíz. De acuerdo con este autor, la desaparición de Procampo implicaría una disminución de 2,858 millones de toneladas en la producción de maíz, y un aumento en las importaciones por la misma cantidad, en relación con los niveles promedio observados en el periodo 1994-1996.

Por la menor producción (18'658,000 toneladas), las importaciones de maíz serían mayores en $40.5 \%$, en relación con las compras externas observadas en el modelo base. Estos resultados indican que sin Procampo, $34.8 \%$ del consumo se abastecería con importaciones, y el restante $65.2 \%$ con producción doméstica.

En el cuadro 2 se presenta el cambio en los excedentes al productor y consumidor, y se puede observar que el bienestar es mayor con el modelo base (que considera la existencia de Procampo). Sin éste, los excedentes al consumidor, al productor y total serían menores en 3,940, 17,248 y 21,188 millones de pesos, respectivamente, en relación con los niveles 
Cuadro 1

Validación del modelo de maíz, 2005/2007

\begin{tabular}{|c|c|c|c|c|}
\hline \multirow[t]{2}{*}{ Región } & $\begin{array}{c}\text { Datos observados } \\
\text { en } 2005 / 2007\end{array}$ & $\begin{array}{c}\text { Modelo base } \\
\text { Procampo existe }\end{array}$ & Diferencia & \multirow[t]{2}{*}{$\begin{array}{c}\text { Diferencia } \\
(\%)\end{array}$} \\
\hline & \multicolumn{3}{|c|}{ Miles de toneladas } & \\
\hline \multicolumn{5}{|l|}{ Consumo } \\
\hline Chiapas & 803 & 826 & 23 & 2.9 \\
\hline Jalisco & 4,678 & 4,785 & 106 & 2.3 \\
\hline Centro & 7,869 & 7,928 & 59 & 0.8 \\
\hline Guanajuato y Michoacán & 2,443 & 2,517 & 74 & 3.0 \\
\hline Sinaloa & 2,826 & 2,890 & 65 & 2.3 \\
\hline Golfo & 2,603 & 2,611 & 8 & 0.3 \\
\hline Oaxaca y Guerrero & 1,241 & 1,281 & 39 & 3.2 \\
\hline Norte & 3,340 & 3,392 & 52 & 1.6 \\
\hline Noreste & 1,657 & 1,669 & 12 & 0.8 \\
\hline Península & 1,146 & 1,148 & 2 & 0.2 \\
\hline Total & 28,606 & 29,047 & 441 & 1.5 \\
\hline \multicolumn{5}{|l|}{ Producción } \\
\hline Chiapas & 1,435 & 1,445 & 10 & 0.7 \\
\hline Jalisco & 3,071 & 3,096 & 25 & 0.8 \\
\hline Centro & 3,537 & 3,701 & 165 & 4.7 \\
\hline Guanajuato y Michoacán & 2,618 & 2,604 & -14 & -0.5 \\
\hline Sinaloa & 4,956 & 4,937 & -19 & -0.4 \\
\hline Golfo & 1,152 & 1,243 & 91 & 7.9 \\
\hline Oaxaca y Guerrero & 1,845 & 1,828 & -17 & -0.9 \\
\hline Norte & 1,559 & 1,650 & 91 & 5.8 \\
\hline Noreste & 717 & 786 & 68 & 9.6 \\
\hline Península & 499 & 541 & 43 & 8.5 \\
\hline Total & 21,390 & 21,833 & 443 & 2.1 \\
\hline \multicolumn{5}{|c|}{ Precio promedio al consumidor (pesos por tonelada) } \\
\hline Chiapas & 1,669 & 1,367 & -302 & -18.1 \\
\hline Jalisco & 2,033 & 1,747 & -286 & -14.1 \\
\hline Centro & 1,894 & 1,811 & -83 & -4.4 \\
\hline Guanajuato y Michoacán & 2,055 & 1,676 & -379 & -18.4 \\
\hline Sinaloa & 2,033 & 1,780 & -254 & -12.5 \\
\hline Golfo & 1,724 & 1,734 & 9 & 0.5 \\
\hline Oaxaca y Guerrero & 1,933 & 1,591 & -342 & -17.7 \\
\hline Norte & 1,819 & 1,641 & -178 & -9.8 \\
\hline Noreste & 1,731 & 1,648 & -83 & -4.8 \\
\hline Península & 1,755 & 1,771 & 15 & 0.9 \\
\hline Total & 1,865 & 1,677 & -188 & -10.1 \\
\hline \multicolumn{5}{|l|}{ Importaciones } \\
\hline Total & 7,216 & 7,214 & -2 & -0.03 \\
\hline
\end{tabular}

Fuente: Elaboración propia con base en información obtenida de los resultados de la solución del modelo base. 


\section{Cuadro 2}

Efectos de Procampo en el mercado de maíz

\begin{tabular}{|c|c|c|c|c|c|c|c|}
\hline Región & Modelo & Escena- & Diferen & Diferencia & Escena- & Diferer & Diferenc \\
\hline & $\begin{array}{c}\text { base } \\
1\end{array}$ & $\begin{array}{c}\text { rio } 1^{b} \\
2\end{array}$ & & $\begin{array}{c}(\%) \\
4=3 / 1\end{array}$ & $\begin{array}{c}\text { rio } 2^{c} \\
5\end{array}$ & & $\begin{array}{c}(\%) \\
7=6 / 1\end{array}$ \\
\hline Miles de tonelad & as métrica & & & & & & \\
\hline Consumo & 29,047 & 28,797 & -250 & -0.9 & 29,169 & 123 & 0.4 \\
\hline Animal & 14,573 & 14,464 & -110 & -0.8 & 14,635 & 62 & 0.4 \\
\hline Humano & 11,419 & 11,286 & -132 & -1.2 & 11,478 & 59 & 0.5 \\
\hline Industrial & 3,054 & 3,047 & -8 & -0.3 & 3,057 & 2 & 0.1 \\
\hline Producción & 21,833 & 18,658 & $-3,175$ & -14.5 & 23,451 & 1,618 & 7.4 \\
\hline Blanco & 20,274 & 17,344 & $-2,930$ & -14.5 & 21,774 & 1,500 & 7.4 \\
\hline Amarillo & 559 & 1,314 & -245 & -15.7 & 1,678 & 118 & 7.6 \\
\hline Importaciones & 7,214 & 10,139 & 2,925 & 40.5 & 5,718 & $-1,495$ & -20.7 \\
\hline Blanco & 124 & 124 & 0 & 0.0 & 124 & 0 & 0.0 \\
\hline Amarillo & 7,090 & 10,015 & 2,925 & 41.3 & 5,594 & $-1,495$ & -21.1 \\
\hline Miles de jornales & & & & & & & \\
\hline Empleo de mano & & & & & & & \\
\hline de obra & 128,613 & 116,083 & $-12,531$ & -9.7 & 134,964 & 6,351 & 4.9 \\
\hline Millones de peso & & & & & & & \\
\hline Exced & & & & & & & \\
\hline económico & 295,860 & 274,672 & $-21,188$ & -7.2 & 306,148 & 13,080 & 4.4 \\
\hline Excedente al & & & & & & & \\
\hline consumidor & 267,846 & 263,906 & $-3,940$ & -1.5 & 269,995 & 2,149 & 0.8 \\
\hline Anin & 93,919 & 92,472 & $-1,448$ & -1.5 & 94,732 & 813 & 0.9 \\
\hline Hum & 150,632 & 148,260 & $-2,372$ & -1.6 & 151,931 & 1,299 & 0.9 \\
\hline Industrial & 23,295 & 23,174 & -121 & -0.5 & 23,331 & 36 & 0.2 \\
\hline Excedente al & & & & & & & \\
\hline proc & 28,014 & 10,766 & $-17,248$ & -61.6 & 45 & 1 & 39.0 \\
\hline Blan & 25,923 & 9,864 & $-16,059$ & -61.9 & 36,153 & 10,230 & 39.5 \\
\hline $\mathrm{Am}$ & 2,091 & 902 & $-1,1$ & -56.8 & 2,792 & 701 & 33.5 \\
\hline $\begin{array}{l}\text { Valor de } \\
\text { importaciones }\end{array}$ & 10,850 & 15,260 & 4,411 & 40.7 & 8,624 & $-2,225$ & -20.5 \\
\hline
\end{tabular}

${ }^{a}$ Con Procampo; ${ }^{b} \sin$ Procampo; ${ }^{c}$ considera un aumento de $50 \%$ en el apoyo otorgado por el Programa.

Fuente: Elaboración propia con base en información obtenida de los resultados de la solución del modelo base.

observados en el año de análisis. Lo anterior significa que Procampo incrementó el bienestar de la población en 21,188 millones de pesos, siendo beneficiados fundamentalmente los productores.

Además del mayor excedente económico, la implementación de Procampo permitió el ahorro de divisas por las menores importaciones. Con el modelo base el valor de las importaciones fue de 10,850 millones de pesos, en tanto que en el escenario 1 este concepto ascendió a 15,260 millones de pesos; lo que significa un ahorro de divisas equivalente a 4,411 millones de pesos.

Los cambios en los excedentes al productor y consumidor, sumados al ahorro de divisas por menores importaciones, permiten determinar los beneficios de la política. Debido a que el consumo y la producción fueron 
mayores con Procampo, el excedente económico total fue mayor en 21,188 millones de pesos en el modelo base, en relación con el escenario 1; sumando el excedente económico más el ahorro de divisas, se tiene un beneficio total de 25,599 millones de pesos. Sin considerar los gastos de administración del Programa y suponiendo que el pago benefició a 100\% de los productores de maíz, lo cual significa que la totalidad de la superficie sembrada en el año 2005/2007 fue apoyada con el subsidio, se tiene que el costo de la política ascendió a 8,573 millones de pesos.

Se ha demostrado que los beneficios derivados por aplicar el Programa son sensiblemente mayores a los costos que el gobierno desembolsa para llevarlo a la práctica; sin embargo, aun con Procampo la pérdida de autosuficiencia alimentaria es notoria, sólo una cuarta parte del consumo de maíz depende de las importaciones. Considerando la mediación realizada y la importancia social, económica y política que el maíz representa para los consumidores y productores de México, se recomienda un aumento sustancial en el subsidio.

Los efectos de un aumento de 50\% en el pago de Procampo se presentan en el cuadro 2. Por efectos de la política, la producción y el consumo aumentarían en 1'618,000 y 123,000 toneladas; por la mayor oferta de maíz, el empleo de mano de obra aumentaría en 6’351,000 jornadas de trabajo. Por la mayor producción, las importaciones descenderían en 1'495,000. En el escenario 2, las importaciones abastecerían $19.2 \%$ del consumo total nacional, permitiendo que poco más de $80 \%$ lo hiciera con producción doméstica.

Un mayor subsidio otorgado por Procampo traería efectos positivos en el bienestar de la sociedad, pues aumentarían los excedentes al productor y consumidor. Los efectos en los excedentes económicos provocados por el mayor subsidio son los siguientes: a) un aumento en el excedente al productor de 10,931 millones de pesos derivado principalmente de una mayor producción; $b$ ) un aumento en el excedente al consumidor por 2,149 millones de pesos, que sumados a los cambios en el excedente al productor generan un incremento en el excedente económico total por 13,080 millones de pesos, y c) un ahorro de divisas equivalente a 2,225 millones de pesos.

Estas cifras suman un total de 15,305 millones de pesos, en tanto que el costo de la política ascendería a 4,286 millones de pesos $(50 \%$ del costo con el modelo base). Los beneficios para la sociedad serían notoriamente superiores a los gastos que el gobierno tendría que desembolsar, esto indica una asignación eficiente en el uso de los recursos, aspecto fundamental en el actual escenario de crisis de la economía nacional.

Son varios los argumentos que se podrían dar para justificar un aumento en el subsidio otorgado por medio de Procampo; sin embargo, la 
autosuficiencia alimentaria es el más importante. Un mayor subsidio permitiría depender menos de las importaciones y, por tanto, reducir los riesgos de la volatilidad de los precios internacionales. El aumento en el precio de los combustibles y la incertidumbre en el mercado mundial del petróleo son algunos de los factores que explican la rápida expansión de la industria del etanol en Estados Unidos, derivado fundamentalmente de maíz, por tanto, es casi seguro que el aumento de la demanda origine un incremento del grano en el mercado estadounidense. En este sentido, si el gobierno de México pretende evitar los efectos nocivos que la escasez del grano y el aumento del precio podrían tener sobre la población de más bajos recursos en un futuro cercano, éste debe estimular la producción mediante el otorgamiento de mayores subsidios.

\section{Conclusiones}

La medición de los efectos de Procampo indica que éste ha estimulado el empleo de mano de obra y la producción de maíz, evitando una mayor dependencia alimentaria por las importaciones; sin Procampo, actualmente más de la tercera parte del consumo se abastecería con importaciones. Los beneficios contabilizados a través de los cambios positivos en los excedentes al productor y consumidor, así como el ahorro de divisas fueron sensiblemente superiores a los costos desembolsados para instrumentar la política. Las importaciones de maíz disminuirían de forma notoria, determinando que sólo una quinta parte del consumo se abasteciera con maíz proveniente del exterior, si el subsidio otorgado por medio del Programa aumentara en $50 \%$. Debido a que una mayor dependencia de las importaciones hace vulnerable al mercado doméstico, se recomienda que el gobierno de México mantenga el Programa, e incluso que se analice la posibilidad de aumentar los apoyos que se otorgan en la actualidad.

\section{Bibliografía}

AMS-USDA (Agricultural Marketing Service-United States Department of Agriculture) (2009), "Grain transportation report", <www.ams. usda.gov>, 12 de febrero de 2009.

Bivings, Leigh (1997), "The seasonal and spatial dimensions of sorghum market liberalization in Mexico", American Journal of Agricultural Economics, 79, American Agricultural Economics Association, Iowa, pp. 383-393. 
Boyd, Roy, Khosrow Doroodian y Saquib Abdul-Latif(1993), "The effects of tariff removals on the North American lumber trade", Canadian Journal of Agricultural Economics, 41 (3), Canadian Agricultural Economics Society, Victoria, British Columbia, pp. 311-328.

Cramer, Gail, Eric Wailes y Shangnan Shui (1993), "Impacts of liberalization trade in the world rice market", America Journal of Agricultural Economics, 75, American Agricultural Economics Association, Iowa, pp. 219-226.

Davis, Benjamín (2007), "Procampo: seis desafíos para su rediseño", Primer Foro Procampo: la Nueva Visión de un Viejo Reto, Apoyos y Servicios a la Comercialización Agropecuaria (Aserca), $<$ www.aserca.gob.mx>, 18 de mayo.

DgtTFM (Dirección General de Tarifas, Transporte Ferroviario y Multimodal) (2000), "Competitividad de los servicios de transporte para maíz en México", ponencia presentada en el Foro Cadenas Productivas: Maíz, Colegio de Postgraduados, Montecillo, Estado de México, 17-18 de mayo.

FAPRI (Food and Agricultural Policy Research Institute) (2009), "Elasticities database", <www.fapri.org/tools/elasticity.aspx>, 10 de febrero de 2009.

García-Salazar, José Alberto (2001), "Efecto de Procampo sobre la producción y saldo de comercio exterior de maíz", Agrociencia, 35 (6), Colegio de Postgraduados, Montecillo, Estado de México, pp. 671-683.

García-Salazar, José Alberto y Gary Williams (2004), "Evaluación de la política comercial respecto al mercado de maíz”, El Trimestre Económico, LXXI (281), Fondo de Cultura Económica, México, pp. 169-213.

García-Salazar, José Alberto, Gary Williams y Jaime Enrique Javier Málaga (2005), "Efectos del TLCAN sobre las exportaciones de tomate de México a los Estados Unidos", Fitotecnia Mexicana, 28 (4), Sociedad Mexicana de Fitogenética, Chapingo, Estado de México, pp. 299-309.

García-Salazar, José Alberto, Gabriela Rodríguez-Licea, Abelardo SáenzTorres y Samuel Rebollar-Rebollar (2006), "Políticas para mejo- 
rar la competitividad de la producción de maíz y frijol en México", Fitotecnia Mexicana, 29 (núm. esp., 2), Sociedad Mexicana de Fitogenética, Chapingo, Estado de México, pp. 115-121.

García-Santillán, Arturo (2004), "Un estudio empírico sobre alianza para el campo, Procampo, remesas y financiamiento bancario y su influencia en el saneamiento de las finanzas rurales y producción agropecuaria. El caso de Aguascalientes. Factibilidad de bursatilizar Procampo", tesis de doctorado, Universidad Autónoma de Aguascalientes, Aguascalientes.

GEA (Grupo de Economistas y Asociados) (2005), "Estudio sobre la evaluación del desempeño del Programa de Apoyos Directos al Campo (Procampo): ciclos agrícolas or 2004/2005 y Pv 2005”, Apoyos y Servicios a la Comercialización Agropecuaria (Aserca), <wwwaserca.gob.mx>, México, 15 de octubre de 2008.

González-König, Gabriel y Wodon Quentin (2005), "Do cash transfers to farmers reduce migration? Procampo in Mexico", Working Paper EC200501, Universidad de Guanajuato, <http://economia. ugto.org/WorkingPapers/EC200501.pdf>, 7 de mayo de 2009.

Hernández-Ortiz, Juan y Miguel Ángel Martínez-Damián (2009), “Efectos del cambio de precios de garantía a Procampo en precios al productor, sin incluir efecto de importaciones", Fitotecnia Mexicana, 32 (2), Sociedad Mexicana de Fitogenética, Chapingo, Estado de México, pp. 153-158.

Herrera-Ramos, Mario (2002), "La nueva política social en México y su diseńo institucional: el caso de Procampo", ponencia presentada en el vir Congreso Internacional del CLAD sobre la Reforma del Estado y Administración Pública”, Lisboa, 8-11 de octubre.

INEGI (Instituto Nacional de Estadística, Geografía e Informática) (1995), $X I V$ censo industrial, XI censo comercial y XI censo de servicios, INEGI, Aguascalientes.

INEGI (Instituto Nacional de Estadística, Geografía e Informática) (2008a), "Finanzas públicas e indicadores monetarios bursátiles", $<$ http://dgcnesyp.inegi.org.mx/cgi-win/bdieintsi.exe>, $21 \mathrm{de}$ octubre de 2008. 
INEGI (Instituto Nacional de Estadística, Geografía e Informática) (2008b), "Precios e inflación", <http://dgcnesyp.inegi.org.mx/cgi-win/ bdieintsi.exe>, 23 de octubre de 2008.

INEGI (Instituto Nacional de Estadística, Geografía e Informática) (2008c), "Cuaderno de información oportuna, núm. 432", <http://www. inegi.gob. $m x>, 11$ de febrero de 2008.

Kawaguchi, Tsunemasa, Nobuhiro Susuki y Harry Kaiser (1997), "A spatial equilibrium model for imperfectly competitive milk markets", American Journal of Agricultural Economics, 79, American Agricultural Economics Association, Iowa, pp. 851-859.

Kondo-López, Jorge (2007), "En la búsqueda de un nuevo Procampo", ponencia presentada en el Foro Procampo: La Nueva Visión de un Viejo Reto, Apoyos y Servicios a la Comercialización Agropecuaria (Aserca), <www.aserca.gob.mx>, México, 22 de agosto de 2007.

Labarthe-Carlock, José María (2007), "Subsidios directos al campo ¿perpetuar dependencia o promover emancipación”, ponencia presentada en el Segundo Foro Procampo: La Nueva Visión de un Viejo Reto, Apoyos y Servicios a la Comercialización Agropecuaria (Aserca), <www.aserca.gob.mx>, México, 19 de julio de 2007.

Martínez-Cruz, Guadalupe (2007), "Propuestas de Consucc para incorporar a los pequeños productores y hacer más eficiente un subsidio para elevar la productividad del sector agrícola de México", ponencia presentada en el Foro de Procampo: La Nueva Visión de un Viejo Reto, Apoyos y Servicios a la Comercialización Agropecuaria (Aserca), México, 22 de agosto de 2007.

Presidencia de la República (2001), "Primer Informe de Gobierno de Vicente Fox Quezada, Anexo Estadístico", <http://primer.informe.fox.presidencia.gob.mx/index.php?idseccion=124>, 15 de junio de 2010.

Presidencia de la República (2009a), "Plan Nacional de Desarrollo 20072012", <http://pnd.calderon.presidencia.gob.mx>, 17 de junio de 2009. 
Presidencia de la República (2009b), "Segundo Informe de Gobierno de Felipe Calderón Hinojosa, Anexo Estadístico”, <http:// www. informe.gob.mx>, 12 de mayo de 2009.

Rebollar-Rebollar, Samuel, José Alberto García-Salazar y Gabriela Rodríguez-Licea (2006), "Análisis espacial e intertemporal sobre el almacenamiento de sorgo en México", Ciencia Ergo-Sum, 12 (3), Universidad Autónoma del Estado de México, Toluca, pp. 245-254.

Rosenzweig-Pichardo, Andrés (2007), "Propuestas para reformular Procampo", ponencia presentada en el Segundo Foro Procampo: La Nueva Visión de un Viejo Reto, Apoyos y Servicios a la Comercialización Agropecuaria (Aserca), <www.aserca.gob.mx>, México, 19 de julio de 2007.

Sadoulet, Elisabeth, Alain de Janvry y Benjamín Davis (2001), "Cash transfers programs with income multipliers: Procampo in Mexico", FCDN Discussion Paper 99, Food Consumption and Nutrition Division, International Food Policy Research Institute, Washington.

Sagarpa (Secretaría de Agricultura, Ganadería, Desarrollo Rural, Pesca y Alimentación) (2002), "Lineamientos y mecanismos específicos de operación del subprograma de apoyo a la pignoración de la cosecha de maíz blanco del ciclo otoño-invierno 2001-2002 del estado de Sinaloa", Diario Oficial de la Federación, 23 de mayo de 2002, Secretaría de Gobernación, <www.diariooficial.segob. gob.mx>, México, 27 de febrero de 2009.

Sagarpa (Secretaría de Agricultura, Ganadería, Desarrollo Rural, Pesca y Alimentación) (2007), Situación actual y perspectivas del maiz en México 1996-2012, Sagarpa, México.

SCT (Secretaría de Comunicaciones y Transportes) (2009), "Tarifa de transporte ferroviario", <http://dgtfm.sct.gob.mx/index.php?id= 439>, 15 de junio de 2009.

Scott, John (2007), "Procampo: eficiencia con equidad", ponencia presentada en el Primer Foro Procampo: La Nueva Visión de un Viejo Reto, Apoyos y Servicios a la Comercialización Agropecuaria (Aserca), <www.aserca.gob.mx>,México, 18 de mayo de 2007. 
Serpro (Servicios Profesionales para el Desarrollo Económico, s.c.) (2008), "Evaluación de consistencia y resultados del Programa de Apoyos Directos al Campo (Procampo) para el Ejercicio Fiscal 2007, Apoyos y Servicios a la Comercialización Agropecuaria (Aserca), <www.aserca.gob.mx>, México, 15 de septiembre de 2009.

SIAP-Sagarpa (Servicio de Información Agroalimentaria y Pesquera-Secretaría de Agricultura, Ganadería, Desarrollo Rural, Pesca y Alimentación) (2009a), "Agricultura, producción anual, cierre de la producción agrícola por estado y cultivo", <http://www.siap.sagarpa.gob.mx>, 16 de enero de 2009.

SIAP-Sagarpa (Servicio de Información Agroalimentaria y Pesquera-Secretaría de Agricultura, Ganadería, Desarrollo Rural, Pesca y Alimentación) (2009b), “Agricultura, producción mensual, resumen de la producción agrícola por cultivo, avances de siembras y cosechas", <www.siap.sagarpa.gob.mx>, 17 de enero de 2009.

SNIIM (Sistema Nacional de Información e Integración de Mercados) (2009), "Mercados nacionales, precios de mercado, mercados agrícolas, granos básicos", <www.secofi-sniim.gob.mx/nuevo>, 15 de marzo de 2007.

Takayama, Takashi y George Judge (1971), Spatial and Temporal Price and Allocation Models, North-Holland Publishing Company, Amsterdam.

Tangerman, Stefan (2006), "Políticas agrícolas y el desarrollo de México", en OCDE, Politicas públicas para un mejor desempeño económico, experiencias del mundo para el desarrollo, México 10 años en la OCDE, OCDE, México, pp. 177-201.

Torres-Sandoval, Clemente y José Alberto García-Salazar (2008), "Aplicación de un modelo de equilibrio espacial para determinar la estructura del mercado de frijol en México", Agrociencia, 42 (6), Colegio de Postgraduados, Montecillo, Estado de México, pp. 731-740.

USITC (Unites States International Trade Commission) (2009), "Interactive tariff and trade DataWeb corn: Fas general customs value/ feneral first unit of quantity by HTS number for Mexico", <http:// dataweb.usitc.gov>, 16 de enero de 2009. 
Recibido: 6 de octubre de 2009.

Reenviado: 23 de julio de 2010. Aceptado: 21 de septiembre de 2010.

José Alberto García-Salazar. Es doctor por el Instituto de Socioeconomía, Estadística e Informática del Colegio de Postgraduados, asimismo realizó estudios de licenciatura y maestría en economía agrícola en la Universidad Autónoma Chapingo y el Colegio de Postgraduados. Ha realizado estancias en la Universidad de Texas A\&M y en la Universidad Estatal de Nuevo México. Actualmente es investigador nivel in en el Sistema Nacional de Investigadores (SNI) y es profesor en el Programa de Economía del Colegio de Postgraduados. Sus líneas de investigación actuales son: política agrícola, mercado y comercio nacional e internacional de productos agropecuarios. Entre sus publicaciones destacan: "Políticas para disminuir las importaciones de frijol en México: un análisis por tipo de variedad", Agrociencia, 42 (8), Colegio de Postgraduados, Montecillo, pp. 949-958 (2008); "Tarifas y consumo de agua en la Comarca Lagunera", Región y Sociedad, xx (42), El Colegio de Sonora, Hermosillo, pp. 119-132 (2008); "Aplicación de un modelo de equilibrio espacial para determinar la estructura del mercado del frijol en México", Agrociencia, 42 (6), Colegio de Postgraduados, Montecillo, pp. 731-740 (2008); "Evaluación de la política comercial de México respecto al mercado de maíz", El Trimestre Económico, LXXI (1), 281, Fondo de Cultura Económica, México, pp. 169213 (2004).

Rhonda K. Skaggs. Es doctora en economía por la Universidad Estatal de Utah, asimismo realizó estudios de maestría en economía agrícola y recursos naturales en la Universidad Estatal de Colorado. Actualmente es profesora en el Departamento de Economía Agrícola y Negocios Agrícolas de la Universidad Estatal de Nuevo México. Sus líneas de investigación actuales son: política agrícola y alimentaria, estructura agrícola y futuro del sistema alimentario y agrícola. Entre sus publicaciones destacan: "Water use by agricultural crops and riparian vegetation: an application of remote sensing technology", Journal of Contemporary Water Research and Education, 137, Universities Council on Water Resources, Southern Illinois University, Carbondale, Illinois, pp. 8-13 (2007); "National animal identification and the elephant in the room", Rangelands, 29, 2, Society for Range Management, Wheatridge, Colorado, pp. 60-64; "Ecological services to and from rangelands of the United States", Ecological Economics, 64, International Society for Ecological Economics, Boston, Massachusetts, pp. 261-268 (2007); "Estimating daily net radiation over vegetation canopy through remote sensing and climatic data", Journal of 
Irrigation and Drainage Engineering, 133 (4), American Society of Civil Engineers, Reston, Virginia, pp. 291-297 (2007).

Terry L. Crawford. Es doctor por el Departamento de Economía Aplicada y Administración de la Universidad de Cornell. Actualmente es profesor en el Departamento de Economía Agrícola y Negocios Agrícolas de la Universidad Estatal de Nuevo México. Sus líneas de investigación actuales son: transferencia de tecnología del agua, estudios sobre comercio, investigación y educación en Nuevo México y ganadería y comercio México-Estados Unidos. Entre sus publicaciones destacan: "Forecasting mexican live cattle exports to the United States", Journal of Agricultural and Resource Economics, 26 (2), Western Agricultural Economics Association, Laramie, Wyoming, pp. 567-568 (2001); "Agricultural trade: big business for U.S. and Mexico", Agricultural Outlook, USDA-ERS, 183, United States Department of Agriculture, Washington, pp. 31-34 (1992); "Mexican cattle exports to U.S.: current perspectives", Agricultural Outlook, USDA-ERS, 282, United States Department of Agriculture, Washington, pp. 6-9 (2002). 